\title{
Can dark neutrino interactions phase out the Hubble tension?
}

\author{
Subhajit Ghosh $\odot,{ }^{*}$ Rishi Khatri, ${ }^{\dagger}$ and Tuhin S. Roy ${ }^{\ddagger}$ \\ Department of Theoretical Physics, Tata Institute of Fundamental Research, Mumbai 400005, India
}

(Received 30 August 2019; revised 3 April 2020; accepted 5 November 2020; published 29 December 2020)

\begin{abstract}
New interactions of neutrinos can stop them from free streaming even after the weak interaction freezeout. This results in a phase shift in the cosmic microwave background (CMB) acoustic peaks which can alleviate the Hubble tension. In addition, the perturbations in neutrinos do not decay away on horizon entry and contribute to metric perturbation enhancing the matter power spectrum. We demonstrate that this acoustic phase shift can be achieved using new interactions of standard left-handed neutrinos with dark matter without changing the number of effective relativistic degrees of freedom. Using Planck CMB and the WiggleZ galaxy survey $\left(k \leq 0.12 \mathrm{~h} \mathrm{Mpc}^{-1}\right)$ data, we demonstrate that in this model the Hubble tension reduces to approximately $2.1 \sigma$. Our model predicts potentially observable modifications of the CMB $B$ modes and the matter power spectrum that can be observed in future datasets.
\end{abstract}

DOI: 10.1103/PhysRevD.102.123544

\section{INTRODUCTION}

The values of the Hubble constant $\left(H_{0}\right)$ inferred from cosmic microwave background (CMB) anisotropies (67.5土 $\left.0.6 \mathrm{~km} \mathrm{~s}^{-1} \mathrm{Mpc}^{-1}[1,2]\right)$ and baryon acoustic oscillations (BAO) measurements (66.98 $\pm 1.18 \mathrm{~km} \mathrm{~s}^{-1} \mathrm{Mpc}^{-1}$ [3-8]) are significantly smaller than the measurements from observations of the nearby Universe using the distance ladder (74.03 $\pm 1.42 \mathrm{~km} \mathrm{~s}^{-1} \mathrm{Mpc}^{-1}$ [9-11]). The gravitational lensing time delay measurements in multiply imaged quasar systems which are independent of the cosmic distance ladder also gives a higher value $\left(72.5_{-2.3}^{+2.1} \mathrm{~km} \mathrm{~s}^{-1} \mathrm{Mpc}^{-1}[12,13]\right)$. Recently, an independent calibration of distance ladder without using the cepheids but using the tip of the red giant branch gives a value of Hubble $H_{0}=69.8 \pm 0.8$ (stat) \pm 1.7 (sys), which is in between the Planck and cepheid based distance ladder values [14]; see also Refs. [15,16]. This tension, calculated using Gaussian error bars, between the Planck CMB and local Hubble measurement stands at approximately $4 \sigma[2,11]$. Increasingly, this tension is being seen as a hint of physics beyond the $\Lambda \mathrm{CDM}^{1}$ cosmology [17-53], rather than a manifestation of possible systematics in the local distance ladder [54-62].

\footnotetext{
subhajit@theory.tifr.res.in

${ }^{\dagger}$ khatri@ theory.tifr.res.in

*tuhin@theory.tifr.res.in

${ }^{1}$ In $\Lambda \mathrm{CDM}, \Lambda$ is the cosmological constant and CDM stands for cold dark matter.
}

Published by the American Physical Society under the terms of the Creative Commons Attribution 4.0 International license. Further distribution of this work must maintain attribution to the author(s) and the published article's title, journal citation, and DOI. Funded by SCOAP ${ }^{3}$.
The spectacular success of the standard models of cosmology and particle physics in describing all cosmological and particle physics observables, however, makes the task of explaining the Hubble tension from new physics (NP) rather nontrivial. Particularly in this context, if the CMB data are to be reinterpreted with NP, the peaks and troughs of the power spectra must match data at least as well as the $\Lambda$ CDM parametrization of the big bang cosmology. The locations of acoustic peaks $[63,64]$ in CMB data approximately correspond to the extrema of the cosine function characterizing the photon temperature transfer function, $\cos \left(k r_{*}+\phi\right)$, where $k$ denotes the comoving wave number, $r_{*}$ is the comoving sound horizon at recombination, and $\phi$ is the phase shift with contribution $(\phi>0)$ from free-streaming neutrinos in $\Lambda \mathrm{CDM}$ cosmology [65]. The peak positions correspond to the wave numbers $k_{\text {peak }}$, which satisfy $k_{\text {peak }} r_{*}=m \pi-\phi$, where $m \geq 1$ is an integer. The corresponding observed CMB peak multipoles $\left(\ell_{\text {peak }}\right)$ are given by

$$
\begin{aligned}
\ell_{\text {peak }} & \approx k_{\text {peak }} D_{\mathrm{A}}=(m \pi-\phi) \frac{D_{\mathrm{A}}}{r_{*}}, \quad \text { where } \\
D_{\mathrm{A}} & =\int_{0}^{z_{*}} d z \frac{1}{H(z)}, \quad r_{*}=\int_{z_{*}}^{\infty} d z \frac{c_{s}(z)}{H(z)},
\end{aligned}
$$

$c_{s}(z)$ is the speed of sound in the baryon-photon plasma, $H(z)$ is the Hubble parameter, and $D_{\mathrm{A}}$ is the comoving angular diameter distance to the redshift of recombination $z_{*}$. Finding a solution to the Hubble tension requires keeping $\ell_{\text {peak }}$ fixed while increasing $H_{0}$.

We see from Eq. (2) that we can modify the late-time evolution of the Universe, i.e., modify $H(z)$ for $z<z_{*}$, in such a way that $D_{\mathrm{A}}$ remains unchanged but $H_{0} \equiv H(0)$ is 

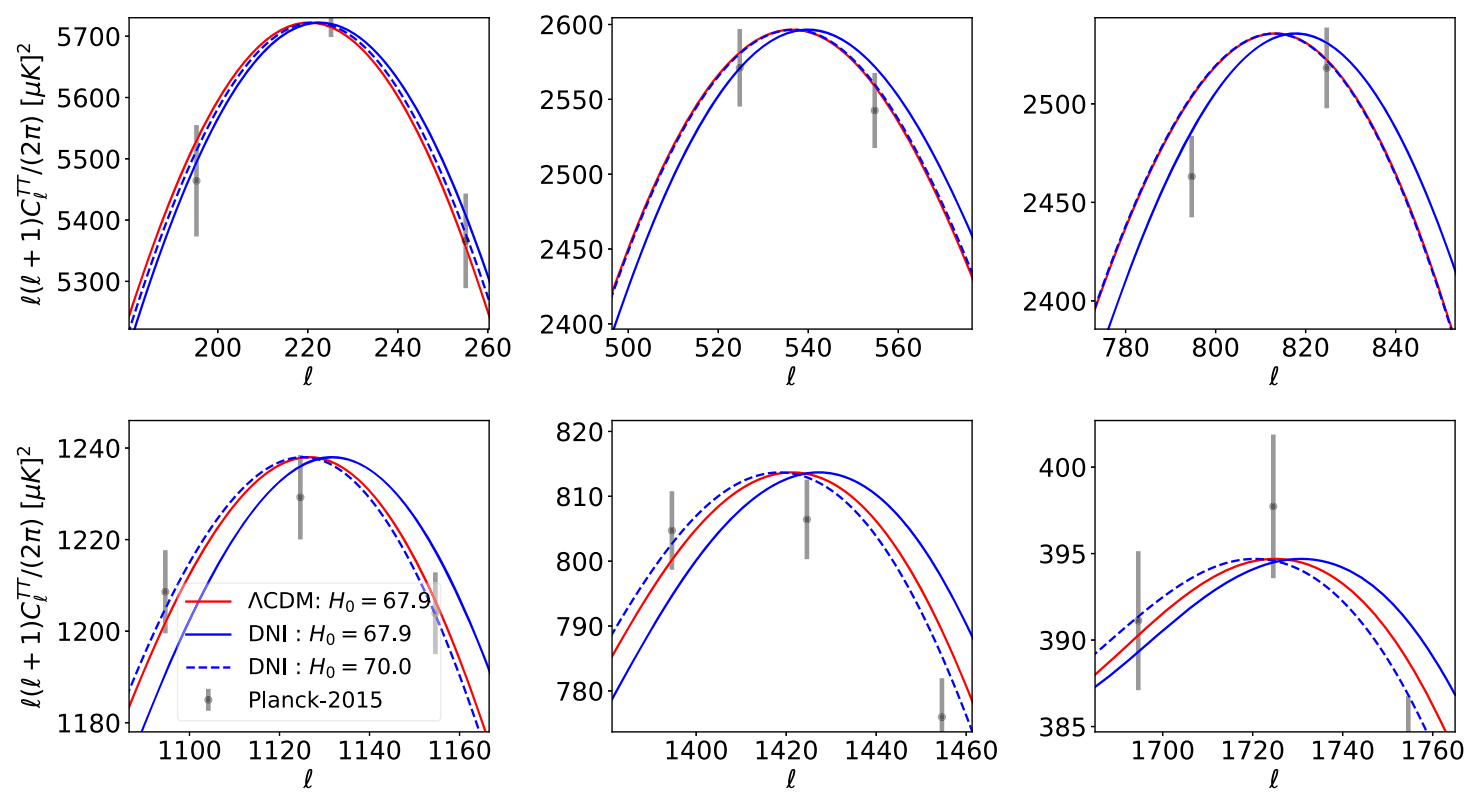

FIG. 1. CMB temperature (TT) power spectrum around first six acoustic peaks. The leftmost solid red line is the best-fit Planck [87] temperature power spectrum with a best-fit value of $H_{0}=67.9 \mathrm{~km} \mathrm{~s}^{-1} \mathrm{Mpc}^{-1}$. Introducing DNI, keeping all other cosmological parameters fixed, moves all peaks to the right/higher $\ell$ with larger shift for higher $\ell$ peaks (rightmost solid blue curves). However, DNI with higher $H_{0}$ brings the peaks back to the original positions (dashed blue). The amplitudes of DNI power spectra for each peak is adjusted so that the peak height is the same as the $\Lambda$ CDM. Also shown as points with error bars is the binned Planck power spectrum.

pushed higher, to reconcile $\mathrm{CMB} / \mathrm{BAO}$ or acoustic $H_{0}$ with local $H_{0} \quad[17,21,22,25-28,30,31,33,36-38,40-44,48,51]$. Since in these solutions the early expansion history of the Universe $\left[H(z)\right.$ for $z>z_{*}$ ] is unchanged, $r_{*}$ remains unaltered. Therefore, $\ell_{\text {peak }}$ remains unchanged from the observed $\Lambda \mathrm{CDM}$ values. A second class of proposals rely on altering the cosmology before radiation domination, i.e., $H(z)$ for $z \gg z_{*}$. These solutions change $r_{*}$, while at the same time keeping $r_{*} / D_{\mathrm{A}}$ fixed [18-20,23,24,29,32, $34,35,39,45-47,49,50,52]$. All of the solutions that have been proposed so far to alleviate the Hubble tension fall into the above two classes and, in particular, keep the acoustic scale at recombination $\theta_{*}=r_{*} / D_{\mathrm{A}}$ fixed even after accommodating a larger Hubble constant.

\section{UNDOING NEUTRINO PHASE-SHIFT}

In this paper, we propose a new class of solutions in which NP solves the Hubble tension by inducing changes in the phase shift $\phi$. These solutions are characterized by acoustic scales $\theta_{*}$ which are different from the acoustic scale in the $\Lambda \mathrm{CDM}$ model. To understand the nature of NP that can accommodate a larger $H_{0}$, let us consider a flat $\Lambda \mathrm{CDM}$ cosmology, with the Hubble parameter given by $H(z)^{2}=H_{0}^{2}\left[\Omega_{\mathrm{m}}(1+z)^{3}+\Omega_{\mathrm{r}}(1+z)^{4}+\left(1-\Omega_{\mathrm{m}}-\Omega_{\mathrm{r}}\right)\right]$, where $\Omega_{i}$ are the ratios of physical energy densities $\left(\rho_{i}\right)$ to the critical energy density today and $i=m, r$ for total nonrelativistic matter and total radiation, respectively. To separate out the effect of changing $H_{0}$, let us keep the physical energy densities of matter and radiation, $\Omega_{\mathrm{m}} H_{0}^{2}$ and $\Omega_{\mathrm{r}} H_{0}^{2}$, fixed. A change $H_{0}^{2} \rightarrow H_{0}^{2}+\delta\left(H_{0}^{2}\right)$ then implies $H(z)^{2} \rightarrow H(z)^{2}+\delta\left(H_{0}^{2}\right)^{2}$ This constant shift in $H(z)$ is only important at low redshifts and becomes unimportant at high redshifts, when $H(z)$ is much larger, and thus has negligible effect on $r_{*}$. Therefore, we see from Eq. (2) that increasing $H_{0}\left(\delta\left(H_{0}^{2}\right)>0\right)$ decreases $D_{\mathrm{A}}\left(\delta D_{\mathrm{A}}<0\right)$. If $\delta D_{\mathrm{A}}$ is to be compensated mostly from the shift in $\phi$ so that $\ell_{\text {peak }}$ remains unaltered, we get from Eq. (2)

$$
\frac{\delta D_{\mathrm{A}}}{D_{\mathrm{A}}}-\frac{\delta \phi_{m}}{m \pi-\phi}=0 \Rightarrow \delta \phi_{m} \approx m \pi \frac{\delta D_{\mathrm{A}}}{D_{\mathrm{A}}},
$$

where we have explicitly used the notation $\delta \phi_{m}$ to refer to the fact that the needed change in phase shift is different for different peaks. We have also used the fact that $\phi \ll \pi$ in the approximate equality. Therefore, if NP needs to accommodate a larger $H_{0}$, it must induce a negative change in the phase shift that increases with $m$.

Incredibly, undoing the phase shift from free-streaming neutrinos in the standard $\Lambda \mathrm{CDM}$ cosmology [65] produces almost exactly the required effect (see Fig. 1). Neutrinos with NP interactions scatter and do not free stream, effectively generating a negative phase shift with respect to $\Lambda \mathrm{CDM}$. Even though there exists a plethora of studies of

\footnotetext{
${ }^{2}$ We note that including a curvature term, $\Omega_{K}(1+z)^{2}$, will result in a redshift-dependent change in the Hubble parameter and therefore cannot compensate for a constant shift in $H(z)^{2}$.
} 
cosmological impacts from nonstandard neutrinos interaction [29,32,66-79], as well as studies of phase shift in the context of varying relativistic degrees of freedom $\left(N_{\text {eff }}\right)$ on the phase shift [80-86], a detailed study of the impact of new neutrino interactions on the scale-dependent acoustic phase shift while keeping $N_{\text {eff }}$ fixed at the standard value of 3.046 has not been performed yet.

\section{DARK NEUTRINO INTERACTIONS}

In this work, we present a simple proof-of-principle model, namely, dark neutrino interactions (DNI), where a component of dark matter interacts with neutrinos stopping them from free streaming. The DNI undo the phase shift induced by the free-streaming neutrinos in the standard model and thus push $H_{0}$ to higher values and yet are safe from all cosmological and particle physics bounds. The necessary feature of this model is a two-component dark matter. Only a small fraction, $f$, of the total dark matter (DM) energy density $\left(\Omega_{\mathrm{DM}}\right)$ is contributed by the component (namely, $\chi$ ) that interacts with neutrinos, the rest being the standard noninteracting cold dark matter (CDM),

$$
\Omega_{\chi}=f \Omega_{\mathrm{DM}}, \quad \Omega_{\mathrm{CDM}}=(1-f) \Omega_{\mathrm{DM}} .
$$

Note that having a small $f$ allows us to evade the constraints typically obtained when all of the dark matter interacts with neutrinos $[67,68,71-74,88]$. The primary ingredients for our model are therefore $(i)$ an interacting dark matter component, $\chi$; $(i i)$ a messenger, $\psi$ (triplet in flavor); and (iii) an electroweak gauge-invariant effective operator involving the Higgs scalar $H$ and the lepton doublet $l$. After $H$ acquires a nonzero vacuum expectation value $(v)$, the effective operator gives marginal interactions among neutrinos, messengers, and dark matter,

$$
\mathcal{L} \supset \frac{1}{\Lambda}\left(H^{\dagger} l\right)(\psi \chi) \Rightarrow \frac{v}{\sqrt{2} \Lambda} \delta_{i j} \nu_{i} \psi_{j} \chi,
$$

where $\Lambda$ is the scale of the effective operator and $i, j$ are flavor indices. For a possible way to generate the interaction in Eq. (4) from a ultraviolet complete model using various symmetries, see Ref. [79]. By construction, neutrinos remain massless, and all three flavors interact with equal strength.

In this work, we focus on cases in which the mediators and dark matter are nearly degenerate in mass. As shown in Ref. [79], this allows the scattering cross section $\left(\sigma_{\chi \nu}\right)$ between the dark matter and neutrinos to become independent of the neutrino temperature $\left(T_{\nu}\right)$. The temperature independence of DNI enables neutrinos to decouple late, undoing the phase shift from free-streaming neutrinos for all the modes entering horizon until recombination. We can write the "differential optical depth", $\dot{\mu} \equiv \mathrm{d} \mu / \mathrm{d} \eta$, for neutrinos in the DNI model as $\dot{\mu}=a n_{\chi} \sigma_{\chi \nu}=a\left(\frac{\rho_{\chi}}{m_{\chi}}\right) \sigma_{\chi \nu}=a f u \rho_{\mathrm{dm}}\left(\frac{\sigma_{\mathrm{th}}}{100 \mathrm{GeV}}\right)$,

where $a$ is the scale factor; $\eta$ is the conformal time; $\sigma_{\text {th }}=$ $6.65 \times 10^{-25} \mathrm{~cm}^{2}$ is the Thomson cross section; $n_{\chi}, \rho_{\chi}, m_{\chi}$ denote the number density, the energy density, and the mass of $\chi$, respectively; and

$$
u \equiv \frac{\sigma_{\chi \nu}}{\sigma_{\mathrm{Th}}} \times \frac{100 \mathrm{GeV}}{m_{\chi}} \simeq\left(\frac{5.5 \mathrm{TeV}}{\Lambda}\right)^{4}\left(\frac{1 \mathrm{MeV}}{m_{\chi}}\right)^{3} .
$$

The perturbation equations for $\nu$ and $\chi$ in DNI are coupled together [71] similar to the perturbations of the baryon-photon system.

We use the notations and perturbation variables in conformal Newtonian gauge as defined in Ref. [89], where $\delta_{i}, \theta_{i}$ stand for overdensity and divergence of fluid velocity respectively for $i$ th species. The total dark matter transfer functions are just the weighted sum of the corresponding perturbation variables of the two dark matter components,

$$
\begin{aligned}
& \delta_{\mathrm{DM}}=f \delta_{\chi}+(1-f) \delta_{\mathrm{CDM}}, \\
& \theta_{\mathrm{DM}}=f \theta_{\chi}+(1-f) \theta_{\mathrm{CDM}} .
\end{aligned}
$$

In the limit, $f \rightarrow 0$, we recover the standard $\Lambda \mathrm{CDM}$ cosmology. In addition, for $f \ll 1$, the total dark matter transfer functions are negligibly different from the CDM transfer functions, and the modifications to the dark matter power spectrum are of order $f^{2}$. Thus, the only difference from the $\Lambda \mathrm{CDM}$ cosmology, when $f \ll 1$, comes from modification of the free streaming of neutrinos. This regime is exactly what we are interested in.

The evolution equations of CDM are unchanged, while the equations of interacting component of DM, $\chi$, get additional terms due to interactions. The neutrino Boltzmann equations are also modified, picking up extra interaction terms,

$$
\begin{gathered}
\dot{\delta}_{\chi}=-\theta_{\chi}+3 \dot{\phi}, \\
\dot{\theta}_{\chi}=-\frac{\dot{a}}{a} \theta_{\chi}+k^{2} \psi-\left(\frac{4 \rho_{\nu}}{3 \rho_{\chi}}\right) \dot{\mu}\left(\theta_{\chi}-\theta_{\nu}\right), \\
\dot{\delta}_{\nu}=-\frac{4}{3} \theta_{\nu}+4 \dot{\phi}, \\
\dot{\theta}_{\nu}=k^{2}\left(\frac{1}{4} \delta_{\nu}-\sigma_{\nu}\right)+k^{2} \psi+\dot{\mu}\left(\theta_{\chi}-\theta_{\nu}\right), \\
\dot{F}_{\nu l}=\frac{k}{(2 l+1)}\left[l F_{\nu l-1}-(l+1) F_{\nu l+1}\right]-\dot{\mu} F_{\nu l},
\end{gathered}
$$

where $k$ is the comoving wave number; the dots represent derivatives with respect to the conformal time; and $\phi$ and $\psi$ 


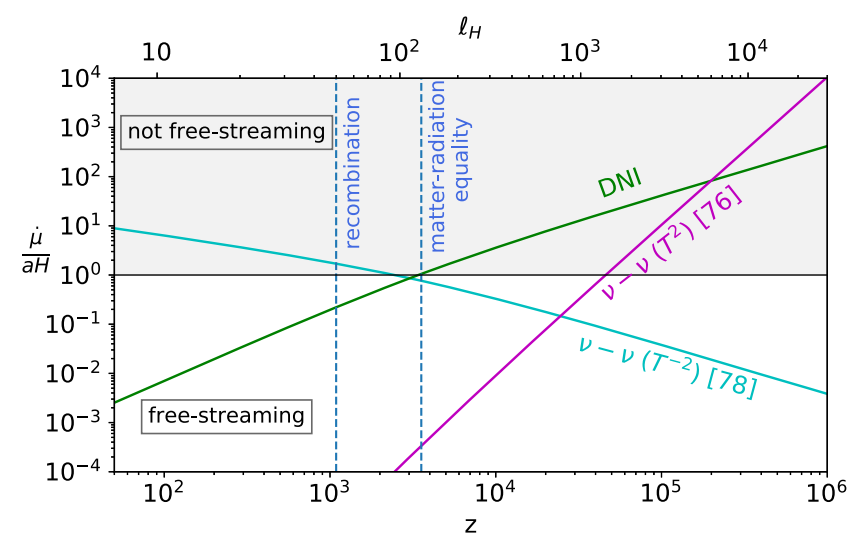

FIG. 2. Comparison of optical depth of neutrinos in DNI $(f u=0.034)$ with models of neutrino self-interaction [76] and [78] having different temperature dependences. The top axis shows the modes $\ell_{H}$ which enter horizon at redshift $z$.

are the Newtonian potentials and $F_{\nu l}$, with $l \geq 2$ being the $l$ th multipole moment of neutrino distribution function. The initial conditions are also modified as the initial anisotropic stress is zero for tightly coupled neutrinos.

We plot the ratio of interaction rate to Hubble rate, $\dot{\mu} /(a H)$, in Fig. 2 for the current upper limits $(f u=0.034)$ for our model derived in this work. For comparison, we also show cases with neutrino self-interaction models [76,78] where cross sections vary as $T_{\nu}^{2}$ and $T_{\nu}^{-2}$. We see from Fig. 2 that with the current upper bounds (fixed $N_{\text {eff }}$ ) on neutrino interactions we can significantly modify the free streaming of neutrinos for all scales which enter horizon before recombination only in the temperature-independent case.

\section{IMPACT ON HUBBLE TENSION}

We have implemented the DNI cosmology in the publicly available code Cosmic Linear Anisotropy Solving System (CLASS) [90]. In DNI cosmology, the modes which enter horizon earlier (higher $\ell$ ) get a larger phase shift (with respect to $\Lambda \mathrm{CDM}$ cosmology) compared to the modes which enter later as shown in Fig. 1 in which we use $f=10^{-3}$, $u=34$. This is because the relative contribution of neutrinos [proportional to $\rho_{\nu} /\left(\rho_{r}+\rho_{m}\right)$, where $\rho_{\nu}$ is the neutrino energy density] to the metric perturbations decreases with time as matter starts to dominate the energy density of the Universe. This is almost exactly the $\ell$ dependence that we need to alleviate the Hubble tension [Eq. (2)]. We show this explicitly in Fig. 3, in which we plot (negative of) the shift in peak positions for the $\mathrm{CMB}$ temperature and $E$-mode polarization angular power spectra $\left[\ell(\ell+1) C_{\ell} /(2 \pi)\right]$ as we change the Hubble constant in $\Lambda \mathrm{CDM}$ cosmology from the best-fit value while keeping other parameters $\left(\Omega_{m} H_{0}^{2}\right.$, etc.) constant. For reference, we show the maximum effect we can get in the curve labeled "No $\nu$-freestreaming" with $\dot{\mu} /(a H) \ggg 1$. We see that the shift in $\ell_{\text {peak }}$ for DNI cosmology, with the current upper bound in temperature
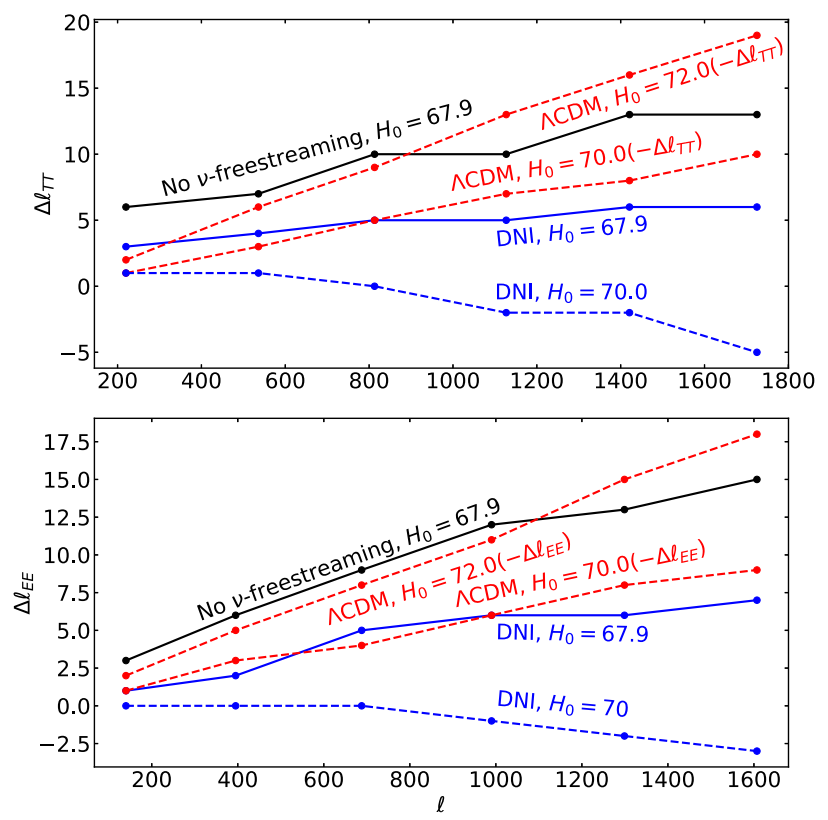

FIG. 3. Shift of the position of peaks of CMB TT $\left(\Delta \ell_{T T}\right)$ and $\operatorname{EE}\left(\Delta \ell_{E E}\right)$ spectrum in $\Lambda \mathrm{CDM}$ and $\mathrm{DNI}$ cosmologies $\left(f=10^{-3}, u=34\right)$ with respect to best-fit $\Lambda \mathrm{CDM}$ model with $H_{0}=67.9 \mathrm{~km} / \mathrm{s} / \mathrm{Mpc}$.

independent interactions, is approximately of the same size (but in the opposite direction) as $\Lambda \mathrm{CDM}$ cosmology with $H_{0}=70 \mathrm{~km} / \mathrm{s} / \mathrm{Mpc}$. The scalings in $\ell$ are also similar in both the cases. The small residual peak shifts at high multipoles are within the error bars. Therefore, we expect that the Hubble tension should reduce considerably in a DNI cosmology. We verify this in the DNI curves with $H_{0}=70 \mathrm{~km} / \mathrm{s} / \mathrm{Mpc}$, in which the peak shifts are negligible compared to the best-fit Planck $\Lambda$ CDM cosmology.

We perform a Markov-chain Monte Carlo (MCMC) analysis of the DNI model using publicly available code MONTE-PYTHON [91]. In addition to the $\Lambda$ CDM parameters, we varied $\omega_{\chi}=\Omega_{\chi} h^{2}$ and $u$, where $h \equiv$ $H_{0} /\left(100 \mathrm{~km} \mathrm{~s}^{-1} \mathrm{Mpc}^{-1}\right)$ is the reduced Hubble constant. The fraction $f$ was extracted as a derived parameter, $f=\frac{\omega_{\chi}}{\omega_{\mathrm{CDM}}+\omega_{\chi}}$, where $\omega_{\mathrm{CDM}}=\Omega_{\mathrm{CDM}} h^{2}$. For all the parameters in the MCMC analysis, we satisfy the Gelman-Rubin convergence criterion $R-1<0.1$.

For $\omega_{\chi}$ and $u$, we have used flat priors with no hard prior upper boundaries. The lower prior boundary was set to 0 for physicality. We use the following cosmological datasets: Planck CMB 2015 Low- $\ell$ TEB, High $\ell$ TT EE TE-Plik lite, and CMB lensing T $+\mathrm{P}$ [87] (named "P15") and full shape of Galaxy power spectrum measured by WiggleZ Dark Energy Survey [92]. We have checked that in DNI cosmology the difference when using lite vs full likelihood is insignificant. WiggleZ data are sensitive to the modification of matter power spectrum due to the small fraction of interacting dark matter and the strongly interacting neutrinos. The WiggleZ power spectrum goes up to 

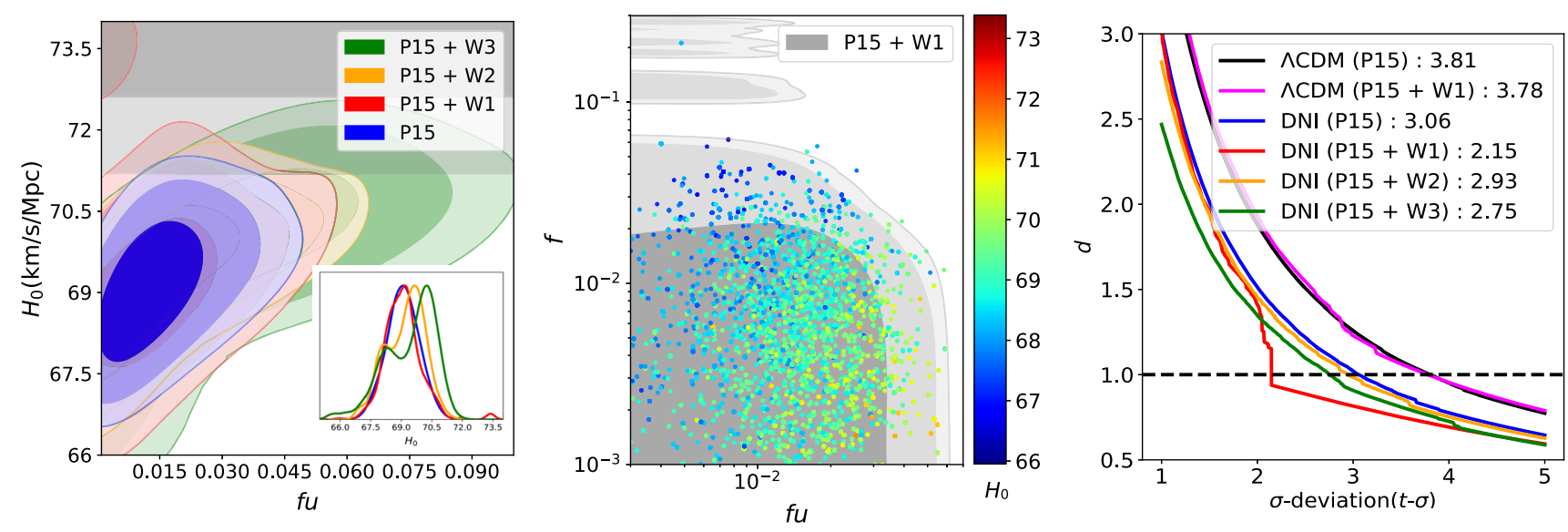

FIG. 4. The left panel shows $1 \sigma, 2 \sigma$, and $3 \sigma$ constraints in DNI and $H_{0}$ for different dataset combinations. The light and dark gray band shows $1 \sigma$ and $2 \sigma$ band for SHOES [11] measurement, respectively. The central panel shows the MCMC samples in the $f-f u$ plane. The right most panel shows calculation of Hubble tension (values given in the legend) taking into account non-Gaussianity of PDFs. The $2 \sigma$ upper limit from $\mathrm{P} 15$ is $f u<0.034$.

$k=0.5 h \mathrm{Mpc}^{-1}$. We have used different $k$ cutoff of the full dataset for three separate analyses and label them W1, W2, and W3 for cutoff $k_{\max }=0.12 h, 0.2 h, 0.3 h \mathrm{Mpc}^{-1}$, respectively. We used the CLASS HALOFIT module [93] to incorporate nonlinear modifications in the power spectrum, since the WiggleZ power spectrum (specifically W2 and W3) goes up to the $k$ modes where these effects are important. Although the HALOFIT is a tested tool for $\Lambda \mathrm{CDM}$ cosmology, since DNI introduce very small changes in the matter power spectrum by construction, the use of HALOFIT is justified in this case.

We note that for the BAO data, it will be incorrect to use just the BAO scale (or $\theta_{*}$ ) extracted from the power spectrum (e.g., Ref. [8] assuming $\Lambda \mathrm{CDM}$ cosmology, available as BAO likelihood modules in public MCMC codes, to constrain any new physics which modifies the phase shift $\phi$ of the acoustic oscillations and allows $\theta_{*}$ to vary from the $\Lambda \mathrm{CDM}$ value. This is the case for us and also for any model with nonstandard $N_{\text {eff }}$, since any new freestreaming relativistic species contributes to $\phi$ in a scaledependent manner.

The marginalized constraints from our MCMC analysis, with two extra parameters $(f, u)$ are shown in Fig. 4. The local measurement from Ref. [11] of $H_{0}=74.03 \pm$ $1.42 \mathrm{~km} \mathrm{~s}^{-1} \mathrm{Mpc}^{-1}$ is shown in gray horizontal bands. There is a clear degeneracy between the neutrino stopping power $(\propto f u)$ and $H_{0}$ which reduces the Hubble tension. We see from the MCMC samples plotted in Fig. 4 (center) that stronger neutrino interaction favors higher $H_{0}$. The two-dimensional contours clearly indicate that data prefer small $f\left(\lesssim 10^{-2}\right)$ and, as discussed above, for small $f$ the results (limits) become independent of the value of $f$ [Eq. (7)]. These are general features of DNI which are present in all the datasets we have analyzed.

The one-dimensional probability distribution functions (PDFs) shown in the inset of Fig. 4 (left) are highly
non-Gaussian. To quantify the tension between nonGaussian PDFs, we define a quantity $d=\left(H_{1}-H_{2}\right)$ / $\sqrt{\sigma_{1}(t)^{2}+\sigma_{2}(t)^{2}}$, where $H_{1}, H_{2}$ are two $H_{0}$ measurements and $\sigma_{1}(t), \sigma_{2}(t)$ are the corresponding " $t-\sigma$ " upper or lower limits. For a Gaussian PDF, $\sigma(t)=t \sigma_{G}$, where $\sigma_{G}$ is the Gaussian $1 \sigma$ error. We use Gaussian error bar for the local $H_{0}$ measurement and plot the quantity $d$ in Fig. 4 (right). The tension is then given by the value of $t$ where $d=1$. Our definition is equivalent to the usual definition of tension in the Gaussian case. We see that for $\Lambda$ CDM the tension is at $3.8 \sigma$, which reduces to less than or approximately equal to $3 \sigma$ in DNI cosmology. The small secondary peak for the "P15 $+\mathrm{W} 1$ " dataset within the $3 \sigma$ region results in a jump in $d$ and reduction in tension to $2.1 \sigma$. We note that the effect of neutrino interactions on the matter power spectrum is not just a phase shift in BAO. The matter power spectrum is also enhanced because the perturbations in neutrinos do not decay away completely on horizon entry in DNI cosmology and contribute to metric perturbations. Both the phase shift in the CMB and the modifications of the matter power spectrum help reduce the tension to $2.1 \sigma$.

In Table I, we present results of a MCMC analysis of DNI cosmology for fixed $f=10^{-3}$, only varying $u$ along with the $\Lambda \mathrm{CDM}$ parameters, ${ }^{3}$ where we also include the local measurement of $H_{0}$ (SH0ES Collaboration [11]). With respect to $\Lambda$ CDM, $\chi^{2}$ reduces by 9 in DNI with one extra parameter $u$. The best-fit value of the Hubble constant turns out to be $H_{0}=70.4$. Note that, as argued before, this increase in $H_{0}$ is associated with a decrease in $D_{A}$, which in turn gets compensated mostly from a change in $\phi$.

\footnotetext{
${ }^{3}$ We varied the energy density of total DM $\omega_{\text {DM }}$ and set the $\mathrm{CDM}$ energy density $\omega_{\mathrm{CDM}}=(1-f) \times \omega_{\mathrm{DM}}$ and interacting DM energy density $\omega_{\chi}=f \times \omega_{\text {DM }}$.
} 
TABLE I. Parameter table for different dataset combinations with fixed $f=10^{-3}$ for DNI. Best-fit values are indicated by "bf". We also show the baryon density $\left(\omega_{b} \equiv \Omega_{b} h^{2}\right)$, total dark matter density $\left(\omega_{\mathrm{DM}} \equiv \Omega_{\mathrm{DM}} h^{2}\right)$, and magnitude of matter power spectrum on $8 h^{-1} \mathrm{Mpc}$ scale $\left(\sigma_{8}\right)$.

\begin{tabular}{|c|c|c|c|c|}
\hline & \multicolumn{2}{|c|}{$\mathrm{P} 15+\mathrm{W} 1+\mathrm{SH} 0 \mathrm{ES}$} & \multicolumn{2}{|c|}{$\mathrm{P} 15+\mathrm{W} 1$} \\
\hline & $\Lambda \mathrm{CDM}$ & DNI & $\Lambda \mathrm{CDM}$ & DNI \\
\hline$H_{0}(\mathrm{~km} / \mathrm{s} / \mathrm{Mpc})(\mathrm{bf})$ & $68.89_{-0.59}^{+0.58}(68.86)$ & $70.25_{-0.61}^{+0.63}(70.37)$ & $68.01_{-0.6}^{+0.58}(68.08)$ & $69.39_{-0.68}^{+0.69}(69.31)$ \\
\hline$f u(\mathrm{bf})$ & 0 & $0.02321_{-0.012}^{+0.0065}(0.01874)$ & 0 & $0.01744_{-0.011}^{+0.0062}(0.01567)$ \\
\hline $100 \omega_{b}$ & $2.243_{-0.015}^{+0.015}$ & $2.251_{-0.015}^{+0.015}$ & $2.226_{-0.016}^{+0.015}$ & $2.238_{-0.015}^{+0.015}$ \\
\hline$\omega_{\mathrm{DM}}$ & $0.1176_{-0.0013}^{+0.0013}$ & $0.1181_{-0.0013}^{+0.0013}$ & $0.1194_{-0.0013}^{+0.0013}$ & $0.1195_{-0.0013}^{+0.0013}$ \\
\hline $\ln 10^{10} A_{s}$ & $3.07_{-0.025}^{+0.024}$ & $3.005_{-0.026}^{+0.025}$ & $3.052_{-0.025}^{+0.017}$ & $2.998_{-0.027}^{+0.021}$ \\
\hline$n_{s}$ & $0.9709_{-0.0046}^{+0.025}$ & $0.9492_{-0.0048}^{+0.000}$ & $0.966_{-0.0045}^{+0.0043}$ & $0.9467_{-0.0051}^{+0.021}$ \\
\hline$\sigma_{8}$ & $0.8283_{-0.009}^{+0.0046}$ & $0.831_{-0.0092}^{+0.0091}$ & $0.826_{-0.0087}^{+0.00072}$ & $0.8308_{-0}^{+0.00087}$ \\
\hline $100 \theta_{*}$ & $1.04201_{-0.00030}^{+0.00030}$ & $1.04643_{-0.00078}^{+0.00094}(+14.7 \sigma)$ & $1.04183_{-0.00029}^{+0.00031}$ & $1.04573_{-0.00087}^{+0.00125}(+13 \sigma)$ \\
\hline bf & 1.04205 & $1.04614(+0.4 \%)$ & 1.04188 & $1.04587(+0.4 \%)$ \\
\hline$r_{*}(\mathrm{Mpc}), \mathrm{bf}$ & 145.07 & $144.93(-0.1 \%)$ & 144.81 & $144.52(-0.2 \%)$ \\
\hline$D_{\mathrm{A}}(\mathrm{Mpc}), \mathrm{bf}$ & 12.78 & $12.71(-0.5 \%)$ & 12.75 & $12.68(-0.6 \%)$ \\
\hline$\Delta \chi^{2}$ & 0 & -9.08 & 0 & -2.42 \\
\hline
\end{tabular}

Therefore, the best fit for DNI cosmology is characterized by a $\theta_{*}$, which is approximately $15 \sigma$ away from that of $\Lambda \mathrm{CDM}$. There is, however, a small change in $r_{*}$, which roughly compensates approximately $20 \%$ change in $D_{A}$. Interestingly, DNI cosmology is a slightly better fit to the P15 + W1 datasets than the $\Lambda \mathrm{CDM}$ cosmology, and $H_{0}$ tension is reduced to $2.9 \sigma$. Since $f \ll 1$, the modification of neutrino free streaming alone results in a higher $H_{0}$ and the better fit of the data. In particular, the modification of the dark matter power spectrum due to the effect of radiation pressure of neutrinos on dark matter is not important for $f \ll 1$.

We show the posterior distribution of $f u$ for fixed $f\left(=10^{-3}\right)$ DNI cosmology (see Table I) in Fig. 5 (left).
DNI cosmology prefers nonzero dark neutrino interactions at greater than or approximately equal to $3 \sigma$ in order to reconcile the local Hubble measurements with the CMB and high redshift $(0.2 \lesssim z \lesssim 0.8)$ large scale structure data. The dashed lines show the $3 \sigma$ upper and lower limits of $f u$. Even without the SHOES data, a nonzero interaction is preferred; however, a zero interaction is not ruled out as the posterior extends to the $f u=0$ boundary. When we include SHOES data, the $f u=0$ point is outside the $3 \sigma$ lower limit. This can be also be seen in the right panel of Fig. 5, in which we plot the $x \sigma$ lower limit vs $x$. This curve flattens out when the PDF starts falling sharply for the without-SHOES case as we hit the boundary. Thus, we conclude that nonzero interactions are demanded for
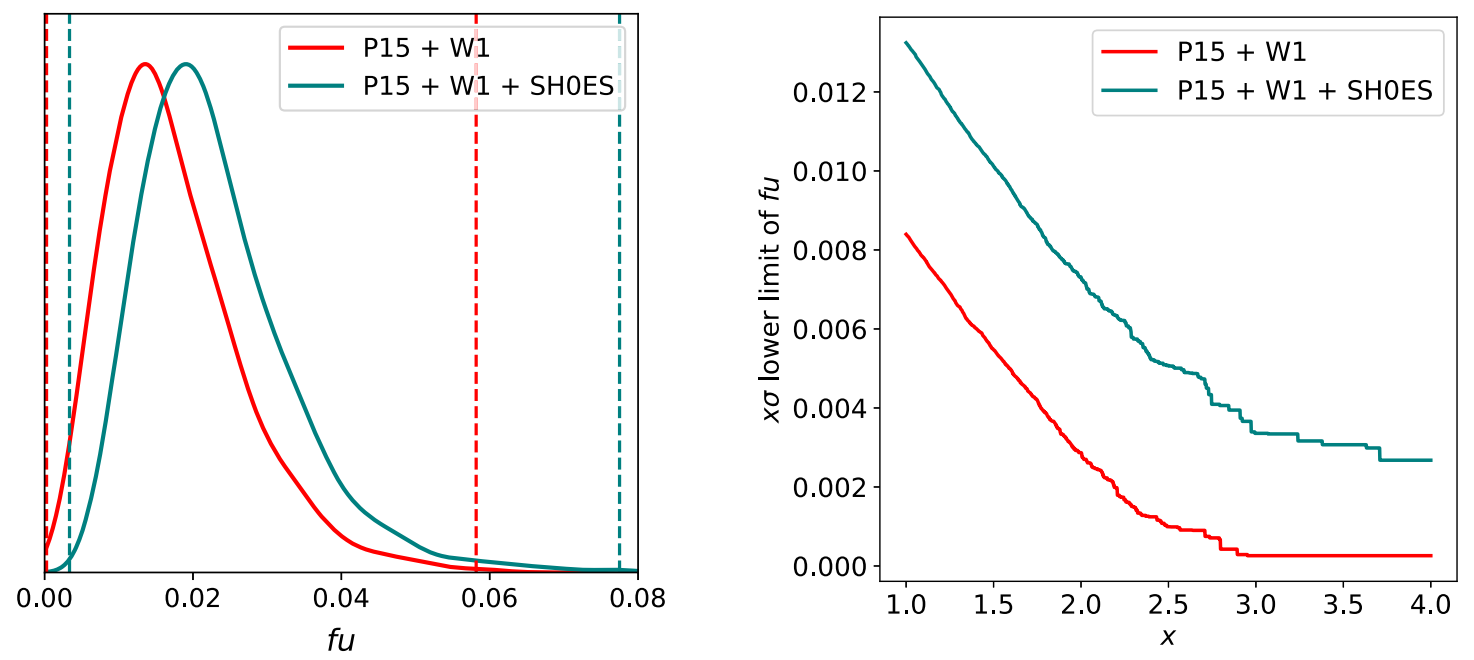

FIG. 5. Left: $1 \mathrm{D}$ posterior for $f u$ for fixed $f$ DNI cosmology (see Table I). The dashed vertical lines marks the $3 \sigma$ upper and lower limits for the corresponding dataset. Right: the $x \sigma$ lower limit on $f u$ plotted against $x$. It can be seen from both the plots that $f u=0$ is excluded at more than $3 \sigma$ when we include SHOES data. 

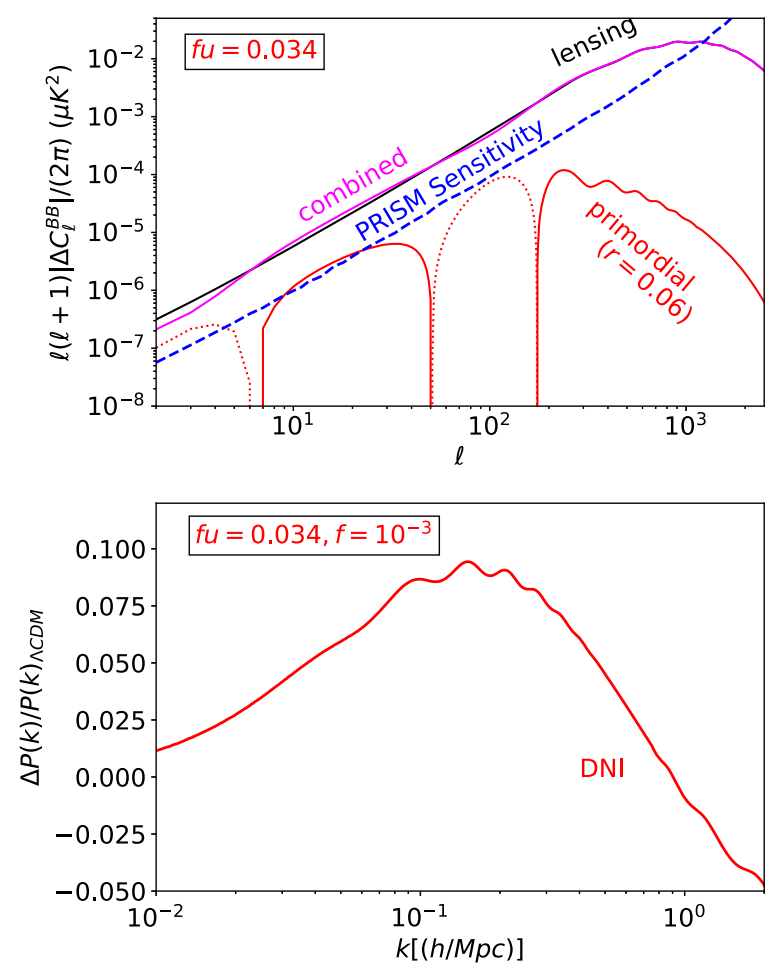

FIG. 6. Absolute change in CMB $B$ modes [primordial (red), lensing (black), and combined (pink)] from $\Lambda$ CDM due to DNI is shown in the top panel. The solid (dotted) lines represent enhancement (suppression) of power. The blue dashed line shows the sensitivity of PRISM [96]. The bottom panel shows the fractional change in matter power spectrum with respect to $\Lambda \mathrm{CDM}$ cosmology.

reconciliation of SHOES data with Planck and WiggleZ. For clarity, we also show the posterior distributions of all the parameters in Fig. 6 in Appendix. The shift in $\theta_{*}$ and $H_{0}$ is clearly visible in the figure. The other parameters having significant shift are $n_{\mathrm{s}}$ and $A_{\mathrm{s}}$.

We see in Table I that the best fit $f u \approx 2 \times 10^{-2}$ requires $u \approx 20$. For $m_{\chi} \lesssim 1 \mathrm{MeV}$, we find the scale of the effective operator to be $\Lambda \gtrsim 2.5 \mathrm{TeV}$ from Eq. (6). For this high a $\Lambda$, we do not expect any significant constraint from particle physics.

The gravity of new neutrino interactions modifies the $B$-mode CMB power spectrum [79] and matter power spectrum as shown in Fig. 6. We compare in Fig. 6 (top) the modification of $B$ modes for tensor-to-scalar ratio $r=0.06$ $[1,94,95]$ with the sensitivity of the proposed experiment Polarized Radiation Imaging and Spectroscopy Mission (PRISM) [96]. This effect, in principle, can be detected if $r$ is close to the current upper limit $[1,94,95]$ with a future PRISM-like experiment [96-100]. In DNI cosmology, the perturbations in neutrinos do not decay away and influence the matter perturbations gravitationally. This results in the enhancement of the matter power spectrum as shown in Fig. 6 (bottom). Of course, there are shifts in the BAO peaks which are analogous to the shifts in the CMB acoustic peaks, as discussed before. The effects in the matter power spectrum are much stronger compared to the CMB $B$ mode. The future large scale structure surveys [101-104] will greatly improve the accuracy of matter power spectrum measurements achieving approximately $1 \%$ accuracy [104] and will therefore be able probe the DNI cosmology.

\section{CONCLUSIONS}

In this work, we have proposed a qualitatively new framework that ameliorates the Hubble tension by primarily using the phase shift in the acoustic oscillations of the primordial plasma. Amazingly, this framework undoes the neutrino induced phase shift of $\Lambda \mathrm{CDM}$, generates the shift in the acoustic peaks of CMB with the required scale dependence, and pushes the acoustic $H_{0}$ higher toward the locally measured value of $H_{0}$. We note that the BAOs are the imprints of the same acoustic oscillations of the primordial plasma in the matter power spectrum $[63,105]$ and therefore any phase shift or the lack of it would be carried over to the BAOs also. Using the Planck CMB and WiggleZ (W1) datasets along with SHOES, we find that new interactions of neutrinos are preferred and no interaction $(f u=0)$ is disfavored at greater than or approximately equal to $3 \sigma$ (Fig. 5). We therefore might have found evidence of new interactions of neutrinos in the Hubble tension. Our modified version of CLASS used in this paper is made publicly available at https://github.com/ subhajitghosh-phy/CLASS_DNI.

\section{ACKNOWLEDGMENTS}

This work was supported by Science and Engineering Research Board (SERB), Government of India, Grants No. ECR/2015/000078 and No. ECR/2015/000196. This work was also supported by Max-Planck-Gesellschaft funded Max Planck partner group between Tata Institute of Fundamental Research, Mumbai, and Max-PlanckInstitut für Astrophysik, Garching. The PDF plots and MCMC analysis were done using the public PYTHON package GETDIST [106]. This work used computational facility of Department of Theoretical Physics, Tata Institute of Fundamental Research. We thank Basudeb Dasgupta for useful comments. We acknowledge support of the Department of Atomic Energy, Government of India, under Project No. 12-R\&D-TFR-5.02-0200.

\section{APPENDIX: TRIANGULAR PLOT FOR FIXED $f$ DNI}

In Fig. 7, we show the plots of one- and two-dimensional posteriors of all the parameters for fixed- $f\left(f=10^{-3}\right) \mathrm{DNI}$ analysis both $\mathrm{P} 15+\mathrm{W} 1$ and $\mathrm{P} 15+\mathrm{W} 1+\mathrm{SH} 0 \mathrm{ES}$ analysis corresponding to Table I. 


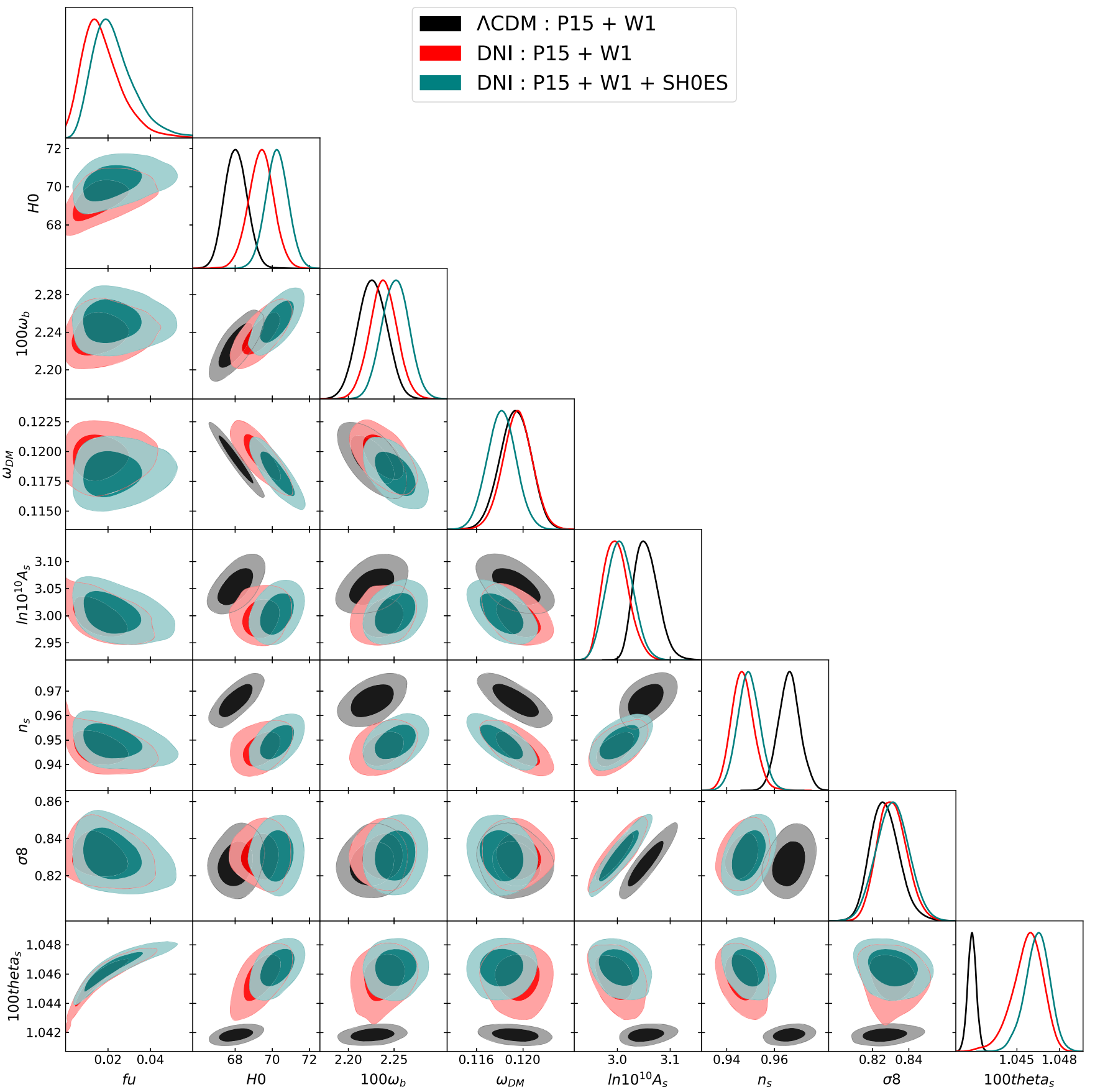

FIG. 7. Triangular plot for $\Lambda \mathrm{CDM}$ and DNI cosmologies (with $f=10^{-3}$ ) corresponding to Table I for $\mathrm{P} 15+\mathrm{W} 1$ and $\mathrm{P} 15+\mathrm{W} 1+$ SHOES dataset. 
[1] P. A. R. Ade et al. (Planck Collaboration), Planck 2015 results. XIII. Cosmological parameters, Astron. Astrophys. 594, A13 (2016).

[2] N. Aghanim et al. (Planck Collaboration), Planck 2018 results. VI. Cosmological parameters, Astron. Astrophys. 641, A6 (2020).

[3] F. Beutler, C. Blake, M. Colless, D. Heath Jones, L. Staveley-Smith, L. Campbell, Q. Parker, W. Saunders, and F. Watson, The $6 \mathrm{dF}$ Galaxy survey: Baryon acoustic oscillations and the local Hubble constant, Mon. Not. R. Astron. Soc. 416, 3017 (2011).

[4] A. Font-Ribera et al. (BOSS Collaboration), QuasarLyman $\alpha$ forest cross-correlation from BOSS DR11: Baryon acoustic oscillations, J. Cosmol. Astropart. Phys. 05 (2014) 027.

[5] T. Delubac et al. (BOSS Collaboration), Baryon acoustic oscillations in the Ly $\alpha$ forest of BOSS DR11 quasars, Astron. Astrophys. 574, A59 (2015).

[6] A. J. Ross, L. Samushia, C. Howlett, W. J. Percival, A. Burden, and M. Manera, The clustering of the SDSS DR7 main Galaxy sample-I. A 4 per cent distance measure at $z=0.15$, Mon. Not. R. Astron. Soc. 449, 835 (2015).

[7] G. E. Addison, D. J. Watts, C. L. Bennett, M. Halpern, G. Hinshaw, and J. L. Weiland, Elucidating $\Lambda$ CDM: Impact of Baryon acoustic oscillation measurements on the Hubble constant discrepancy, Astrophys. J. 853, 119 (2018).

[8] S. Alam et al. (BOSS Collaboration), The clustering of galaxies in the completed SDSS-III Baryon oscillation spectroscopic survey: Cosmological analysis of the DR12 galaxy sample, Mon. Not. R. Astron. Soc. 470, 2617 (2017).

[9] A. G. Riess, L. M. Macri, S. L. Hoffmann, D. Scolnic, S. Casertano, A. V. Filippenko, B. E. Tucker, M. J. Reid, D. O. Jones, and J. M. Silverman, A $2.4 \%$ determination of the local value of the Hubble constant, Astrophys. J. 826, 56 (2016).

[10] A. G. Riess et al., Milky Way Cepheid standards for measuring cosmic distances and application to Gaia DR2: Implications for the Hubble constant, Astrophys. J. 861, 126 (2018).

[11] A. G. Riess, S. Casertano, W. Yuan, L. M. Macri, and D. Scolnic, Large magellanic cloud cepheid standards provide a $1 \%$ foundation for the determination of the Hubble constant and stronger evidence for physics beyond LambdaCDM, Astrophys. J. 876, 85 (2019).

[12] V. Bonvin et al., HOLiCOW-V. New COSMOGRAIL time delays of HE 0435-1223: $H_{0}$ to 3.8 per cent precision from strong lensing in a flat $\Lambda \mathrm{CDM}$ model, Mon. Not. R. Astron. Soc. 465, 4914 (2017).

[13] S. Birrer et al., HOLiCOW-IX. Cosmographic analysis of the doubly imaged quasar SDSS $1206+4332$ and a new measurement of the Hubble constant, Mon. Not. R. Astron. Soc. 484, 4726 (2019).

[14] W. L. Freedman et al., The Carnegie-Chicago Hubble program. VIII. An independent determination of the Hubble constant based on the tip of the red Giant branch, Astrophys. J. 882, 34 (2019).

[15] W. Yuan, A. G. Riess, L. M. Macri, S. Casertano, and D. Scolnic, Consistent calibration of the tip of the red Giant branch in the large magellanic cloud on the Hubble space telescope photometric system and implications for the determination of the Hubble constant, Astrophys. J. 886, 61 (2019).

[16] W. L. Freedman, B. F. Madore, T. Hoyt, I. S. Jang, R. Beaton, M. Gyoon Lee, A. Monson, J. Neeley, and J. Rich, Calibration of the Tip of the Red Giant Branch (TRGB), Astrophys. J. 891, 57 (2020).

[17] A. Barreira, B. Li, C. M. Baugh, and S. Pascoli, The observational status of Galileon gravity after Planck, J. Cosmol. Astropart. Phys. 08 (2014) 059.

[18] C. Umiltà, M. Ballardini, F. Finelli, and D. Paoletti, CMB and BAO constraints for an induced gravity dark energy model with a quartic potential, J. Cosmol. Astropart. Phys. 08 (2015) 017.

[19] J. Lesgourgues, G. Marques-Tavares, and M. Schmaltz, Evidence for dark matter interactions in cosmological precision data? J. Cosmol. Astropart. Phys. 02 (2016) 037.

[20] U. Alam, S. Bag, and V. Sahni, Constraining the cosmology of the phantom brane using distance measures, Phys. Rev. D 95, 023524 (2017).

[21] E. Di Valentino, A. Melchiorri, and J. Silk, Reconciling Planck with the local value of $H_{0}$ in extended parameter space, Phys. Lett. B 761, 242 (2016).

[22] Q.-G. Huang and K. Wang, How the dark energy can reconcile Planck with local determination of the Hubble constant, Eur. Phys. J. C 76, 506 (2016).

[23] P. Ko and Y. Tang, Light dark photon and fermionic dark radiation for the Hubble constant and the structure formation, Phys. Lett. B 762, 462 (2016).

[24] T. Karwal and M. Kamionkowski, Dark energy at early times, the Hubble parameter, and the string axiverse, Phys. Rev. D 94, 103523 (2016).

[25] S. Kumar and R. C. Nunes, Probing the interaction between dark matter and dark energy in the presence of massive neutrinos, Phys. Rev. D 94, 123511 (2016).

[26] J. Renk, M. Zumalacárregui, F. Montanari, and A. Barreira, Galileon gravity in light of ISW, CMB, BAO and $\mathrm{H}_{0}$ data, J. Cosmol. Astropart. Phys. 10 (2017) 020.

[27] E. Di Valentino, A. Melchiorri, E. V. Linder, and J. Silk, Constraining dark energy dynamics in extended parameter space, Phys. Rev. D 96, 023523 (2017).

[28] E. Di Valentino, A. Melchiorri, and O. Mena, Can interacting dark energy solve the $H_{0}$ tension?, Phys. Rev. D 96, 043503 (2017).

[29] E. Di Valentino, C. Bøehm, E. Hivon, and F. R. Bouchet, Reducing the $H_{0}$ and $\sigma_{8}$ tensions with dark matter-neutrino interactions, Phys. Rev. D 97, 043513 (2018).

[30] K. Bolejko, Emerging spatial curvature can resolve the tension between high-redshift $\mathrm{CMB}$ and low-redshift distance ladder measurements of the Hubble constant, Phys. Rev. D 97, 103529 (2018).

[31] E. Di Valentino, E. V. Linder, and A. Melchiorri, Vacuum phase transition solves the $H_{0}$ tension, Phys. Rev. D 97, 043528 (2018).

[32] L. Lancaster, F.-Y. Cyr-Racine, L. Knox, and Z. Pan, A tale of two modes: Neutrino free-streaming in the early universe, J. Cosmol. Astropart. Phys. 07 (2017) 033.

[33] N. Khosravi, S. Baghram, N. Afshordi, and N. Altamirano, $H_{0}$ tension as a hint for a transition in gravitational theory, Phys. Rev. D 99, 103526 (2019). 
[34] M. A. Buen-Abad, M. Schmaltz, J. Lesgourgues, and T. Brinckmann, Interacting dark sector and precision cosmology, J. Cosmol. Astropart. Phys. 01 (2018) 008.

[35] F. D'Eramo, R. Z. Ferreira, A. Notari, and J. Luis Bernal, Hot axions and the $H_{0}$ tension, J. Cosmol. Astropart. Phys. 11 (2018) 014.

[36] K. Dutta, Ruchika, A. Roy, A. A. Sen, and M. M. Sheikh-Jabbari, Beyond $\Lambda$ CDM with low and high redshift data: Implications for dark energy, Gen. Relativ. Gravit. 52, 15 (2020).

[37] A. Banihashemi, N. Khosravi, and A.H. Shirazi, Ginzburg-Landau theory of dark energy: A framework to study both temporal and spatial cosmological tensions simultaneously, Phys. Rev. D 99, 083509 (2019).

[38] E. Belgacem, Y. Dirian, S. Foffa, and M. Maggiore, Nonlocal gravity. Conceptual aspects and cosmological predictions, J. Cosmol. Astropart. Phys. 03 (2018) 002.

[39] K. L. Pandey, T. Karwal, and S. Das, Alleviating the $H_{0}$ and $\sigma_{8}$ anomalies with a decaying dark matter model, J. Cosmol. Astropart. Phys. 07 (2020) 026.

[40] P. Agrawal, F.-Y. Cyr-Racine, D. Pinner, and L. Randall, Rock 'n' Roll solutions to the Hubble tension, arXiv:1904 .01016 .

[41] P. Agrawal, G. Obied, and C. Vafa, $H_{0}$ tension, Swampland conjectures and the epoch of fading dark matter, arXiv: 1906.08261.

[42] E. Di Valentino, R. Z. Ferreira, L. Visinelli, and U. Danielsson, Late time transitions in the quintessence field and the $H_{0}$ tension, Phys. Dark Universe 26, 100385 (2019).

[43] H. Desmond, B. Jain, and J. Sakstein, A local resolution of the Hubble tension: The impact of screened fifth forces on the cosmic distance ladder, Phys. Rev. D 100, 043537 (2019).

[44] S. Pan, W. Yang, E. Di Valentino, A. Shafieloo, and S. Chakraborty, Reconciling $H_{0}$ tension in a six parameter space?, J. Cosmol. Astropart. Phys. 06 (2020) 062.

[45] K. Vattis, S. M. Koushiappas, and A. Loeb, Dark matter decaying in the late Universe can relieve the H0 tension, Phys. Rev. D 99, 121302 (2019).

[46] V. Poulin, T. L. Smith, T. Karwal, and M. Kamionkowski, Early Dark Energy Can Resolve The Hubble Tension, Phys. Rev. Lett. 122, 221301 (2019).

[47] M.-X. Lin, M. Raveri, and W. Hu, Phenomenology of modified gravity at recombination, Phys. Rev. D 99, 043514 (2019).

[48] X.-L. Li, A. Shafieloo, V. Sahni, and A. A. Starobinsky, Revisiting metastable dark energy and tensions in the estimation of cosmological parameters, Astrophys. J. 887, 153 (2019).

[49] S. Alexander and E. McDonough, Axion-Dilaton destabilization and the Hubble tension, Phys. Lett. B 797, 134830 (2019).

[50] M.-X. Lin, G. Benevento, W. Hu, and M. Raveri, Acoustic dark energy: Potential conversion of the Hubble tension, Phys. Rev. D 100, 063542 (2019).

[51] E. Di Valentino, A. Melchiorri, O. Mena, and S. Vagnozzi, Interacting dark energy after the latest Planck, DES, and $H_{0}$ measurements: An excellent solution to the $H_{0}$ and cosmic shear tensions, Phys. Dark Universe 30, 100666 (2020).

[52] M. Archidiacono, D. C. Hooper, R. Murgia, S. Bohr, J. Lesgourgues, and M. Viel, Constraining dark matter-Dark radiation interactions with $\mathrm{CMB}, \mathrm{BAO}$, and Lyman- $\alpha$, J. Cosmol. Astropart. Phys. 10 (2019) 055.

[53] L. Knox and M. Millea, The Hubble Hunter's guide, Phys. Rev. D 101, 043533 (2020).

[54] X.-D. Shi and M. S. Turner, Expectations for the difference between local and global measurements of the Hubble constant, Astrophys. J. 493, 519 (1998).

[55] G. Efstathiou, $\mathrm{H}_{0}$ revisited, Mon. Not. R. Astron. Soc. 440, 1138 (2014).

[56] É. Aubourg et al., Cosmological implications of baryon acoustic oscillation measurements, Phys. Rev. D 92, 123516 (2015).

[57] I. Odderskov, S. Hannestad, and T. Haugbølle, On the local variation of the Hubble constant, J. Cosmol. Astropart. Phys. 10 (2014) 028.

[58] E. Macaulay et al. (DES Collaboration), First cosmological results using Type Ia Supernovae from the dark energy survey: Measurement of the Hubble constant, Mon. Not. R. Astron. Soc. 486, 2184 (2019).

[59] K. Aylor, M. Joy, L. Knox, M. Millea, S. Raghunathan, and W. L. Kimmy Wu, Sounds discordant: Classical distance ladder \& $\Lambda \mathrm{CDM}$-based determinations of the cosmological sound horizon, Astrophys. J. 874, 4 (2019).

[60] S. Taubenberger, S. H. Suyu, E. Komatsu, I. Jee, S. Birrer, V. Bonvin, F. Courbin, C. E. Rusu, A. J. Shajib, and K. C. Wong, The Hubble constant determined through an inverse distance ladder including quasar time delays and Type Ia supernovae, Astron. Astrophys. 628, L7 (2019).

[61] W. D'Arcy Kenworthy, D. Scolnic, and A. Riess, The local perspective on the Hubble tension: Local structure does not impact measurement of the Hubble constant, Astrophys. J. 875, 145 (2019).

[62] M. Rameez and S. Sarkar, Is there really a 'Hubble tension'?, arXiv:1911.06456.

[63] R. A. Sunyaev and Y. B. Zeldovich, Small-scale fluctuations of Relic radiation, Astrophys. Space Sci. 7, 3 (1970).

[64] P. J. E. Peebles and J. T. Yu, Primeval adiabatic perturbation in an expanding iniverse, Astrophys. J. 162, 815 (1970).

[65] S. Bashinsky and U. Seljak, Neutrino perturbations in CMB anisotropy and matter clustering, Phys. Rev. D 69, 083002 (2004).

[66] N. F. Bell, E. Pierpaoli, and K. Sigurdson, Cosmological signatures of interacting neutrinos, Phys. Rev. D 73, 063523 (2006).

[67] G. Mangano, A. Melchiorri, P. Serra, A. Cooray, and M. Kamionkowski, Cosmological bounds on dark matterneutrino interactions, Phys. Rev. D 74, 043517 (2006).

[68] P. Serra, F. Zalamea, A. Cooray, G. Mangano, and A. Melchiorri, Constraints on neutrino-Dark matter interactions from cosmic microwave background and large scale structure data, Phys. Rev. D 81, 043507 (2010).

[69] F.-Y. Cyr-Racine and K. Sigurdson, Limits on neutrinoneutrino scattering in the Early Universe, Phys. Rev. D 90, 123533 (2014).

[70] M. Archidiacono and S. Hannestad, Updated constraints on non-standard neutrino interactions from Planck, J. Cosmol. Astropart. Phys. 07 (2014) 046.

[71] R. J. Wilkinson, C. Boehm, and J. Lesgourgues, Constraining dark matter-neutrino interactions using the CMB 
and large-scale structure, J. Cosmol. Astropart. Phys. 05 (2014) 011.

[72] C. Boehm, J. A. Schewtschenko, R. J. Wilkinson, C. M. Baugh, and S. Pascoli, Using the Milky Way satellites to study interactions between cold dark matter and radiation, Mon. Not. R. Astron. Soc. 445, L31 (2014).

[73] B. Bertoni, S. Ipek, D. McKeen, and A. E. Nelson, Constraints and consequences of reducing small scale structure via large dark matter-neutrino interactions, J. High Energy Phys. 04 (2015) 170.

[74] M. Escudero, O. Mena, A. C. Vincent, R. J. Wilkinson, and C. Bœhm, Exploring dark matter microphysics with galaxy surveys, J. Cosmol. Astropart. Phys. 09 (2015) 034.

[75] F. Forastieri, M. Lattanzi, and P. Natoli, Constraints on secret neutrino interactions after Planck, J. Cosmol. Astroppart. Phys. 07 (2015) 014.

[76] I. M. Oldengott, T. Tram, C. Rampf, and Y. Y. Y. Wong, Interacting neutrinos in cosmology: Exact description and constraints, J. Cosmol. Astropart. Phys. 11 (2017) 027.

[77] C. D. Kreisch, F.-Y. Cyr-Racine, and O. Doré, The neutrino puzzle: Anomalies, interactions, and cosmological tensions, Phys. Rev. D 101, 123505 (2020).

[78] F. Forastieri, M. Lattanzi, and P. Natoli, Cosmological constraints on neutrino self-interactions with a light mediator, Phys. Rev. D 100, 103526 (2019).

[79] S. Ghosh, R. Khatri, and T.S. Roy, Dark neutrino interactions make gravitational waves blue, Phys. Rev. D 97, 063529 (2018).

[80] B. Follin, L. Knox, M. Millea, and Z. Pan, First Detection of the Acoustic Oscillation Phase Shift Expected from the Cosmic Neutrino Background, Phys. Rev. Lett. 115, 091301 (2015).

[81] D. Baumann, D. Green, J. Meyers, and B. Wallisch, Phases of new physics in the CMB, J. Cosmol. Astropart. Phys. 01 (2016) 007.

[82] Z. Pan, L. Knox, B. Mulroe, and A. Narimani, Cosmic microwave background acoustic peak locations, Mon. Not. R. Astron. Soc. 459, 2513 (2016).

[83] D. Baumann, D. Green, and M. Zaldarriaga, Phases of new physics in the BAO spectrum, J. Cosmol. Astropart. Phys. 11 (2017) 007.

[84] D. Baumann, D. Green, and B. Wallisch, Searching for light relics with large-scale structure, J. Cosmol. Astropart. Phys. 08 (2018) 029.

[85] G. Choi, C.-T. Chiang, and M. LoVerde, Probing decoupling in dark sectors with the cosmic microwave background, J. Cosmol. Astropart. Phys. 06 (2018) 044.

[86] D. Baumann, F. Beutler, R. Flauger, D. Green, M. Vargas-Magańa, A. Slosar, B. Wallisch, and C. Yèche, First constraint on the neutrino-induced phase shift in the spectrum of baryon acoustic oscillations, Nat. Phys. 15, 465 (2019).

[87] N. Aghanim et al. (Planck Collaboration), Planck 2015 results. XI. CMB power spectra, likelihoods, and robustness of parameters, Astron. Astrophys. 594, A11 (2016).

[88] R. Primulando and P. Uttayarat, Dark matter-neutrino interaction in light of collider and neutrino telescope data, J. High Energy Phys. 06 (2018) 026.
[89] C.-P. Ma and E. Bertschinger, Cosmological perturbation theory in the synchronous and conformal Newtonian gauges, Astrophys. J. 455, 7 (1995).

[90] D. Blas, J. Lesgourgues, and T. Tram, The Cosmic Linear Anisotropy Solving System (CLASS). Part II: Approximation schemes, J. Cosmol. Astropart. Phys. 07 (2011) 034.

[91] B. Audren, J. Lesgourgues, K. Benabed, and S. Prunet, Conservative constraints on early cosmology: an illustration of the Monte Python cosmological parameter inference code, J. Cosmol. Astropart. Phys. 02 (2013) 001.

[92] D. Parkinson et al., The WiggleZ dark energy survey: Final data release and cosmological results, Phys. Rev. D 86, 103518 (2012).

[93] R. E. Smith, J. A. Peacock, A. Jenkins, S. D. M. White, C. S. Frenk, F. R. Pearce, P. A. Thomas, G. Efstathiou, and H. M. P. Couchmann (Virgo Consortium Collaboration), Stable clustering, the halo model and nonlinear cosmological power spectra, Mon. Not. R. Astron. Soc. 341, 1311 (2003).

[94] P. A. R. Ade et al. (BICEP2 and Keck Array Collaborations), BICEP2/Keck Array V: Measurements of B-mode polarization at degree angular scales and $150 \mathrm{GHz}$ by the Keck Array, Astrophys. J. 811, 126 (2015).

[95] P. A. R. Ade et al. (BICEP2 and Keck Array Collaborations), BICEP2/Keck Array x: Constraints on Primordial Gravitational Waves using Planck, WMAP, and New BICEP2/Keck Observations through the 2015 Season, Phys. Rev. Lett. 121, 221301 (2018).

[96] P. Andre et al. (PRISM Collaboration), PRISM (Polarized Radiation Imaging and Spectroscopy Mission): An extended white paper, J. Cosmol. Astropart. Phys. 02 (2014) 006.

[97] F. Finelli et al. (CORE Collaboration), Exploring cosmic origins with CORE: Inflation, J. Cosmol. Astropart. Phys. 04 (2018) 016.

[98] M. Alvarez et al., PICO: Probe of inflation and cosmic origins, arXiv:1908.07495.

[99] K. N. Abazajian et al. (CMB-S4 Collaboration), CMB-S4 science book, first edition, arXiv:1610.02743.

[100] M. Hazumi et al., LiteBIRD: A satellite for the studies of B-mode polarization and inflation from cosmic background radiation detection, J. Low Temp. Phys. 194, 443 (2019).

[101] A. Aghamousa et al. (DESI Collaboration), The DESI experiment part I: Science, targeting, and survey design, arXiv:1611.00036.

[102] L. Amendola et al., Cosmology and fundamental physics with the Euclid satellite, Living Rev. Relativity 21, 2 (2018).

[103] Ž. Ivezić et al. (LSST Collaboration), LSST: From science drivers to reference design and anticipated data products, Astrophys. J. 873, 111 (2019).

[104] R. Laureijs et al., Euclid definition study report, arXiv: 1110.3193.

[105] D. J. Eisenstein and W. Hu, Baryonic features in the matter transfer function, Astrophys. J. 496, 605 (1998).

[106] A. Lewis and S. Bridle, Cosmological parameters from CMB and other data: A Monte Carlo approach, Phys. Rev. D 66, 103511 (2002). 